\title{
Tritium production from ternary fission
}

\author{
O. Serot ${ }^{1, a}$, C. Wagemans ${ }^{2}$, S. Vermote ${ }^{2}$, J. Heyse ${ }^{3, b}$, T. Soldner ${ }^{4}$, P. Geltenbort ${ }^{4}$, I. AlMahamid ${ }^{5,6}$, \\ J. Floyd ${ }^{6}$, and W.W. Lukens ${ }^{6}$ \\ 1 CEA-Cadarache, DEN/DER/SPRC/LEPh, Bât. 230, 13108 Saint-Paul-lez-Durance, France \\ 2 Dept. of Subatomic and Radiation Physics, University of Gent, Proeftuinstraat 86, 9000 Gent, Belgium \\ 3 EC-JRC, Institute for Reference Materials and Measurements, Retieseweg 111, 2440 Geel, Belgium \\ 4 Institut Laue-Langevin, BP. 156, 38042 Grenoble Cedex 9, France \\ 5 Wadsworth Center, New York State Department of Health, Albany, NY 12201, USA \\ 6 Lawrence Berkeley National Laboratory, Berkeley, CA 94720, USA
}

\begin{abstract}
The tritium gas emission in the thermal neutron induced ternary fission of ${ }^{249} \mathrm{Cf}$ has been determined for the first time in a measurement at the intense neutron beam PF1B of the Institut Laue-Langevin in Grenoble (France). A ${ }^{249} \mathrm{Cf}$ sample was mounted in the centre of a vacuum chamber, at a short distance of a $\Delta \mathrm{E}-\mathrm{E}$ telescope which permitted the identification of the ternary particles. The binary fission fragments were detected in a separate run, using only the E detector. From these measurements, the energy distributions and the emission probabilities of the ternary ${ }^{3} \mathrm{H}$ particles emitted in the thermal neutron induced ternary fission of ${ }^{249} \mathrm{Cf}$ could be determined. Based on this result and on measurements published by us recently, we could enlarge the database related to ${ }^{3} \mathrm{H}$ emission probabilities (denoted $t / B$ ) and their energy distributions in particular for the $\mathrm{Cm}$ and $\mathrm{Cf}$ isotopes. The impact of the excitation energy of the fissioning nucleus on the ternary particle emission probability is discussed, comparing for a given compound nucleus, the $t / B$ data obtained from thermal neutron induced fission (excitation energy of the fissioning nucleus $=$ the neutron binding energy) and from spontaneous fission (excitation energy $=$ zero). In addition, semi-empirical relations between $t / B$ data and characteristics of the binary fission process are proposed.
\end{abstract}

\section{Introduction}

Since several years, our group has been involved in a systematic study of the characteristics of ternary $\alpha$ and triton particles emitted during spontaneous fission decays or from thermal neutron induced fission. Search for correlations between ternary particle properties (energy or angular distributions, emission probability...) and binary fission fragment properties (prompt neutron multiplicity, available deformation energy...) can be very useful for our understanding of the ternary fission process. In addition, helium and tritium gas produced in nuclear reactors originate mainly from ternary fission, and data concerning this production are therefore requested by nuclear safety specialists. The goal of the present paper is threefold:

- First, we will shortly describe new experimental results for the ternary triton particles emitted from the ${ }^{249} \mathrm{Cf}\left(\mathrm{n}_{\mathrm{th}}, \mathrm{f}\right)$ reaction. We will explain how these new data influence the ternary triton emission probability in a previous ${ }^{251} \mathrm{Cf}\left(\mathrm{n}_{\mathrm{th}}, \mathrm{f}\right)$ measurement [1].

- Secondly, we present the new database on the ternary triton characteristics which is now completed with our new results on $\mathrm{Cf}$ (present work) and $\mathrm{Cm}$ ([2]) isotopes.

- Lastly, from this enlarged database, systematics in the energy distribution parameters (average energy and width) are discussed. In addition, the influence of the excitation energy on the $\mathrm{t} / \mathrm{B}$ behaviour is studied.

${ }^{a}$ Presenting author, e-mail: olivier.serot@cea.fr

$b$ Present address: SCK-CEN, Boeretang 200, 2400 Mol, Belgium

\section{New results on $\mathrm{Cf}$ isotopes}

\subsection{Ternary tritons emitted from the ${ }^{249} \mathrm{Cf}\left(\mathrm{n}_{\mathrm{th}}, \mathrm{f}\right)$ reaction}

The measurement of the yields and energy distributions of ternary particles emitted from the thermal neutron induced fission of ${ }^{249} \mathrm{Cf}$ has been performed at the PF1B cold neutron guide of the Institut Laue-Langevin in Grenoble (France). The (very rare) ${ }^{249} \mathrm{Cf}$ sample, deposited on a Ti foil, is almost $100 \%$ pure and its mass is $5.8 \mu \mathrm{g}$. The neutron flux at the sample position was about $3.5 \times 10^{9}$ neutrons $/\left(\mathrm{s} . \mathrm{cm}^{2}\right)$. The sample was placed in the centre of a vacuum chamber at an angle of $45^{\circ}$ with the incident neutron beam as shown in figure 1 . Two $\triangle \mathrm{E}-\mathrm{E}$ telescopes, each consisting of a thin $\Delta \mathrm{E}$ and a thick E silicon surface barrier detector, were placed on both sides of the sample at about $4 \mathrm{~cm}$ distance and perpendicular to the beam (see fig. 1). The measurement was performed in 3 steps:

- The first step consists in the determination of the binary fission counting rate. The sample was placed to face the $\triangle \mathrm{E}-\mathrm{E}$ telescope as shown by the straight line in figure 1 .

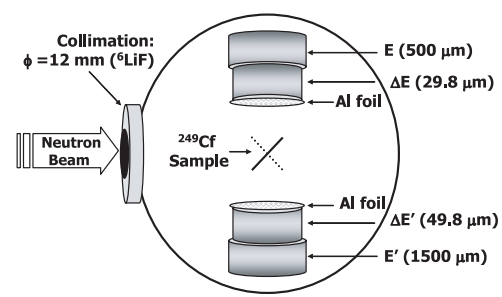

Fig. 1. Experimental setup used for the ${ }^{249} \mathrm{Cf}\left(\mathrm{n}_{\mathrm{th}}, \mathrm{f}\right)$ measurement. 

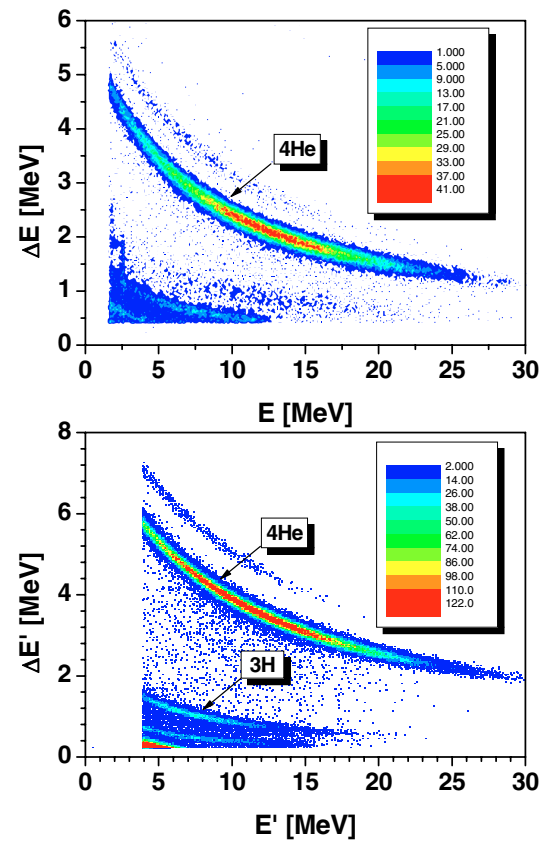

Fig. 2. Two dimensional plots obtained from the $\Delta \mathrm{E}-\mathrm{E}$ (top) and $\Delta \mathrm{E}^{\prime}$-E' (bottom) telescopes.

The $\mathrm{Al}$ foil is removed and the $\Delta \mathrm{E}$ detector is replaced by an empty dummy (with exactly the same dimensions as the $\Delta \mathrm{E}$ detector). Binary fission events are detected by the $\mathrm{E}$ detector and the binary fission counting rate $\left(\mathrm{N}_{B F}\right)$ can then be determined.

- The second step consists in the measurement of the ternary alpha particles (also called the Long Range Alpha particles). It is done using the $\Delta \mathrm{E}-\mathrm{E}$ telescope. A $30 \mu \mathrm{m} \mathrm{Al}$ foil was put in front of the telescope to stop the fission fragments and the ${ }^{249} \mathrm{Cf}$ decay $\alpha$ 's. A $29.8 \mu \mathrm{m} \Delta \mathrm{E}$ and $500 \mu \mathrm{m}$ E telescope allows a good separation between LRA particles and the background as can be seen in the top of figure 2. After the selection of the ternary alpha's, their energy (given by $\Delta E+E$ ) is corrected for the energy loss in the sample and in the $\mathrm{Al}$ foil as well. Performing a Gaussian fit on this corrected spectrum allows the determination of the average energy and the Full Width of Half Maximum (FWHM) of the energy distribution. The area of the fit gives the ternary alpha counting rate (noted $\mathrm{N}_{L R A}$ ). The ternary alpha emission probability (noted LRA/B) is given by the ratio $\mathrm{N}_{L R A} / \mathrm{N}_{B F}$.

- For the third step, the sample was turned over an angle of $90^{\circ}$ in order to place it in front of the $\Delta \mathrm{E}^{\prime}-\mathrm{E}^{\prime}$ telescope (dashed line in fig. 1). Again, a $30 \mu \mathrm{m} \mathrm{Al}$ foil covered the telescope. A $49.8 \mu \mathrm{m} \Delta \mathrm{E}^{\prime}$ and $1500 \mu \mathrm{m} \mathrm{E}^{\prime}$ telescope was used to measure the ternary triton and alpha yields simultaneously. The use of a thicker $\Delta \mathrm{E}$ ' detector (compared to the $\Delta \mathrm{E}$ detector) allows a better separation between the ternary particles, but has the disadvantage of raising the energy threshold at which particles reach the E' detector (see bottom of fig. 2). As in the second step, the energy distribution for both LRA and triton particles can be determined and their counting rate can then be deduced. Figure 3 shows the triton energy distribution. We found: $\mathrm{N}_{L R A}^{\prime}=(0.876 \pm 0.027) \alpha / \mathrm{s}$ and $\mathrm{N}_{t}^{\prime}=(0.069 \pm$

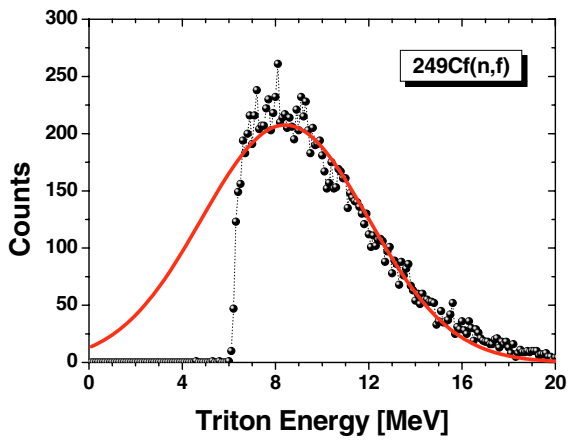

Fig. 3. Energy spectrum of the ternary triton emitted from the ${ }^{249} \mathrm{Cf}\left(\mathrm{n}_{\mathrm{th}}, \mathrm{f}\right)$ reaction.

0.009) $t / \mathrm{s}$. The ratio between triton and alpha particles is therefore: $\mathrm{N}_{t}^{\prime} / \mathrm{N}_{L R A}^{\prime}=(7.9 \pm 1.2) \%$. Combining results obtained from the two last steps, the ternary triton emission probability (noted $\mathrm{t} / \mathrm{B}$ ) can be deduced: $\mathrm{t} / \mathrm{B}=\left(\mathrm{N}_{t}^{\prime} / \mathrm{N}_{L R A}^{\prime}\right) \times$ $\left(\mathrm{N}_{L R A} / \mathrm{N}_{B F}\right)$.

Results relative to the triton particles are included in table 1, where the uncertainties are a combination of both systematic and statistical errors.

\subsection{Ternary tritons emitted from the ${ }^{251} \mathrm{Cf}\left(\mathrm{n}_{\mathrm{th}}, \mathrm{f}\right)$ reaction}

In May 2003, we have carried out the same type of measurement but with a ${ }^{251} \mathrm{Cf}$ sample ([1]). Due to the isotopic composition of the sample, the contributions from the ${ }^{249} \mathrm{Cf}\left(\mathrm{n}_{\mathrm{th}}, \mathrm{f}\right)$ reaction and from the ${ }^{250} \mathrm{Cf}(\mathrm{sf})$ and ${ }^{252} \mathrm{Cf}(\mathrm{sf})$ decays had to be taken into account. Unfortunately at that time, the ternary particle emission probabilities for the ${ }^{249} \mathrm{Cf}\left(\mathrm{n}_{\mathrm{th}}, \mathrm{f}\right)$ reaction were not known and therefore their contribution could not be removed properly. This situation has now changed and the analysis of this experiment can be revisited. In May 2003, the measured binary fission counting rate due to the ${ }^{249} \mathrm{Cf}\left(\mathrm{n}_{\mathrm{th}}, \mathrm{f}\right)$ and ${ }^{251} \mathrm{Cf}\left(\mathrm{n}_{\mathrm{th}}, \mathrm{f}\right)$ reactions was: $\left(N_{n f}^{B F}=1346.2 \pm 10.0 \mathrm{BF} / \mathrm{s}\right)$. This counting rate can be written as:

$$
N_{n f}^{B F}=\epsilon \Phi \times\left(\sigma_{n f}^{249} N^{249}+\sigma_{n f}^{251} N^{251}\right)
$$

where $\epsilon$ is the solid angle of the detection and $\Phi$ the neutron flux; $N^{249}$ and $N^{251}$ represent the number of atoms of ${ }^{249} \mathrm{Cf}$ and ${ }^{251} \mathrm{Cf}$ respectively; $\sigma_{n f}^{249}$ and $\sigma_{n f}^{251}$ are the fission cross sections at the average energy of the neutron beam, i.e., $\overline{E_{n}}=5.4 \mathrm{meV}$. Similarly, the measured ternary triton counting rate was: $N_{n f}^{3}=(0.316 \pm 0.054) s^{-1}$, which can also be expressed as:

$$
N_{n f}^{3 H}=\epsilon \Phi \times\left((t / B)_{249 C f} \sigma_{n f}^{249} N^{249}+(t / B)_{251 C f} \sigma_{n f}^{251} N^{251}\right) .
$$

Combining equations (1) and (2), the ternary triton emission probability, $(\mathrm{t} / \mathrm{B})_{251 C f}$, can be determined and the results are also mentioned in table 1 .

\section{New database for ternary triton particles}

An updated database for the ${ }^{3} \mathrm{H}$ emission probabilities and their energy distributions is reported in table 1. This 
Table 1. Survey of the triton emission probability (t/B) and their energy distribution characteristics (average energy $\bar{E}$ and FWHM). The average prompt neutron multiplicities data for $\left(\mathrm{n}_{\mathrm{th}}, \mathrm{f}\right)$ reactions $\left(\overline{v_{p}}\left(\mathrm{E}_{e x c}\right)\right)$ and for $(\mathrm{sf})$ decays $\left(\overline{v_{p}}\left(\mathrm{E}_{e x c}=0\right)\right)$ are mentioned.

\begin{tabular}{|c|c|c|c|c|c|c|c|}
\hline Reaction & $\mathrm{E}_{e x c}[\mathrm{MeV}]$ & $\overline{\overline{v_{p}}}\left(\mathrm{E}_{\text {exc }}\right)$ & $\overline{v_{p}}\left(\mathrm{E}_{e x c}=0\right)$ & $\mathrm{t} / \mathrm{B} \times 10^{4}$ & $\bar{E}[\mathrm{MeV}]$ & FWHM [MeV] & Reference \\
\hline${ }^{244} \mathrm{Cm}(\mathrm{sf})$ & 0 & $2.690 \pm 0.008$ & $2.690 \pm 0.008$ & $1.91 \pm 0.41$ & $7.6 \pm 0.2$ (fixed) & $7.36 \pm 0.80$ & [2]+Present work \\
\hline${ }^{246} \mathrm{Cm}(\mathrm{sf})$ & 0 & $2.941 \pm 0.008$ & $2.941 \pm 0.008$ & $1.72 \pm 0.24$ & $8.05 \pm 0.34$ & $7.77 \pm 0.47$ & [1] \\
\hline${ }^{248} \mathrm{Cm}(\mathrm{sf})$ & 0 & $3.134 \pm 0.006$ & $3.134 \pm 0.006$ & $1.79 \pm 0.21$ & $8.77 \pm 0.16$ & $7.85 \pm 0.21$ & [3] \\
\hline${ }^{250} \mathrm{Cf}(\mathrm{sf})$ & 0 & $3.511 \pm 0.037$ & $3.511 \pm 0.037$ & $2.70 \pm 0.50$ & $6.9 \pm 0.4$ & $10.2 \pm 1.1$ & [8] \\
\hline${ }^{252} \mathrm{Cf}(\mathrm{sf})$ & 0 & $3.768 \pm 0.005$ & $3.768 \pm 0.005$ & $2.43 \pm 0.17$ & $7.7 \pm 0.4$ & $8.2 \pm 0.9$ & [8] \\
\hline${ }^{256} \mathrm{Fm}(\mathrm{sf})$ & 0 & $3.621 \pm 0.057$ & $3.621 \pm 0.057$ & $3.90 \pm 0.50$ & $6.1 \pm 0.7$ & $10.1 \pm 2.6$ & [8] \\
\hline${ }^{229} \mathrm{Th}\left(\mathrm{n}_{\mathrm{th}}, \mathrm{f}\right)$ & 6.794 & $2.080 \pm 0.020$ & $1.353 \pm 0.136$ & $0.85 \pm 0.15$ & & & [7] \\
\hline${ }^{233} \mathrm{U}\left(\mathrm{n}_{\mathrm{th}}, \mathrm{f}\right)$ & 6.844 & $2.498 \pm 0.004$ & $1.766 \pm 0.177$ & $1.14 \pm 0.05$ & $8.4 \pm 0.2$ & $6.8 \pm 0.3$ & [7] \\
\hline${ }^{235} \mathrm{U}\left(\mathrm{n}_{\mathrm{th}}, \mathrm{f}\right)$ & 6.545 & $2.437 \pm 0.004$ & $1.900 \pm 0.050$ & $1.08 \pm 0.04$ & $8.4 \pm 0.2$ & $6.7 \pm 0.3$ & [7] \\
\hline${ }^{239} \mathrm{Pu}\left(\mathrm{n}_{\mathrm{th}}, \mathrm{f}\right)$ & 6.534 & $2.882 \pm 0.005$ & $2.142 \pm 0.005$ & $1.42 \pm 0.07$ & $8.4 \pm 0.2$ & $7.3 \pm 0.3$ & [7] \\
\hline${ }^{241} \mathrm{Pu}\left(\mathrm{n}_{\mathrm{th}}, \mathrm{f}\right)$ & 6.309 & $2.946 \pm 0.006$ & $2.134 \pm 0.006$ & $1.41 \pm 0.06$ & $8.4 \pm 0.1$ & $6.9 \pm 0.2$ & [9] \\
\hline${ }^{241} \mathrm{Am}\left(\mathrm{n}_{\mathrm{th}}, \mathrm{f}\right)$ & 5.538 & $3.121 \pm 0.023$ & $2.528 \pm 0.254$ & $1.65 \pm 0.10$ & & & [6] \\
\hline${ }^{243} \mathrm{Cm}\left(\mathrm{n}_{\mathrm{th}}, \mathrm{f}\right)$ & 6.801 & $3.422 \pm 0.045$ & $2.690 \pm 0.008$ & $1.77 \pm 0.39$ & $7.8 \pm 0.2$ (fixed) & $7.9 \pm 0.2($ fixed $)$ & {$[2]+$ Present work } \\
\hline${ }^{245} \mathrm{Cm}\left(\mathrm{n}_{\mathrm{th}}, \mathrm{f}\right)$ & 6.458 & $3.825 \pm 0.032$ & $2.941 \pm 0.008$ & $1.85 \pm 0.10$ & $8.4 \pm 0.2$ & $7.76 \pm 0.10$ & [4] \\
\hline${ }^{247} \mathrm{Cm}\left(\mathrm{n}_{\mathrm{th}}, \mathrm{f}\right)$ & 6.213 & $3.790 \pm 0.150$ & $3.134 \pm 0.006$ & $1.84 \pm 0.11$ & $8.55 \pm 0.27$ & $7.52 \pm 0.33$ & [5] \\
\hline${ }^{249} \mathrm{Cf}\left(\mathrm{n}_{\mathrm{th}}, \mathrm{f}\right)$ & 6.625 & $4.080 \pm 0.040$ & $3.511 \pm 0.037$ & $2.20 \pm 0.35$ & $8.41 \pm 0.17$ & $8.41 \pm 0.99$ & Present Work \\
\hline${ }^{251} \mathrm{Cf}\left(\mathrm{n}_{\mathrm{th}}, \mathrm{f}\right)$ & 6.172 & $4.100 \pm 0.500$ & $3.768 \pm 0.005$ & $2.37 \pm 0.58$ & $8.55 \pm 0.59$ & $8.05 \pm 0.91$ & [1]+Present Work \\
\hline
\end{tabular}

database is that of ref. [3] completed with our latest results on ${ }^{249} \mathrm{Cf}\left(\mathrm{n}_{\mathrm{th}}, \mathrm{f}\right)$ and ${ }^{251} \mathrm{Cf}\left(\mathrm{n}_{\mathrm{th}}, \mathrm{f}\right)$. Results on ${ }^{243} \mathrm{Cm}\left(\mathrm{n}_{\mathrm{th}}, \mathrm{f}\right)$ and ${ }^{244} \mathrm{Cm}$ (sf) are those published in ref. [2], slightly modified in order to take the background properly into account. So, for spontaneous fission, results on ${ }^{244} \mathrm{Cm}$ up to ${ }^{256} \mathrm{Fm}$ nuclides are available, while the neutron induced fission data cover target nuclei between ${ }^{229} \mathrm{Th}$ and ${ }^{251} \mathrm{Cf}$.

\section{Discussion}

\subsection{Triton energy distributions}

In figure 4, the average energy (top) and the FWHM (bottom) of the triton energy distribution are plotted, using data from spontaneous fission decays and thermal neutron induced fission reactions. This figure confirms that within the uncertainties, the most probable triton energy remains remarkably constant, except for ${ }^{250} \mathrm{Cf}(\mathrm{sf})$ and ${ }^{256} \mathrm{Fm}(\mathrm{sf})$. We found an average value (weighted by the uncertainties) of $(8.24 \pm 0.06) \mathrm{MeV}$. For the FWHM, a clear increase with the fissility parameter $\left(Z_{C N}^{2} / A_{C N}\right)$ of the compound nucleus can be seen. This behavior (which was already observed in the past [7]) is quite well explained from trajectory calculations. We found: FWHM = $-(18.62 \pm 5.40)+(0.71 \pm 0.15) Z_{C N}^{2} / A_{C N}$.

\subsection{Influence of the excitation energy}

In order to study the impact of the excitation energy (brought by capture of a neutron) on the ternary triton emission probability, we have compared this probability for the same fissioning nucleus at zero excitation energy (spontaneous fission) and at an energy corresponding to the neutron binding energy (thermal neutron induced fission). With the new available database (see table 1), five compound nuclei can be studied: ${ }^{244} \mathrm{Cm} ;{ }^{246} \mathrm{Cm} ;{ }^{248} \mathrm{Cm} ;{ }^{250} \mathrm{Cf}$ and ${ }^{252} \mathrm{Cf}$. In figure 5, the (t/B) ratio between $\left(\mathrm{n}_{\mathrm{th}}, \mathrm{f}\right)$ and $(\mathrm{sf})$ for the same compound nucleus

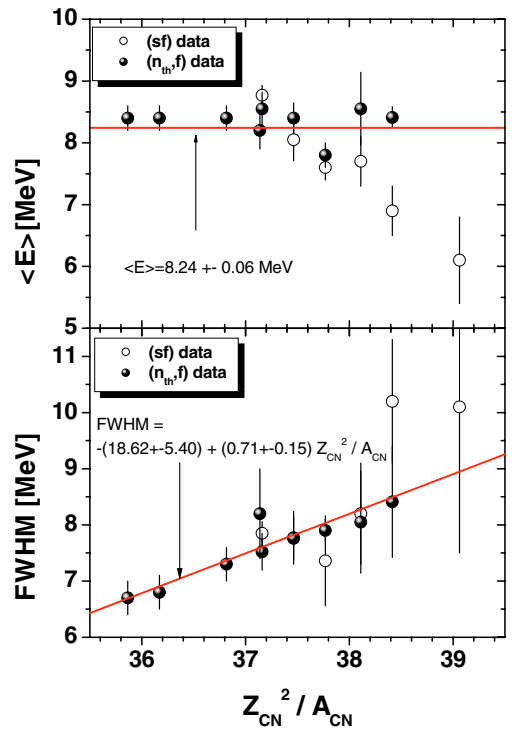

Fig. 4. Average energy (top) and Full Width at Half Maximum (bottom) of the triton energy distributions.

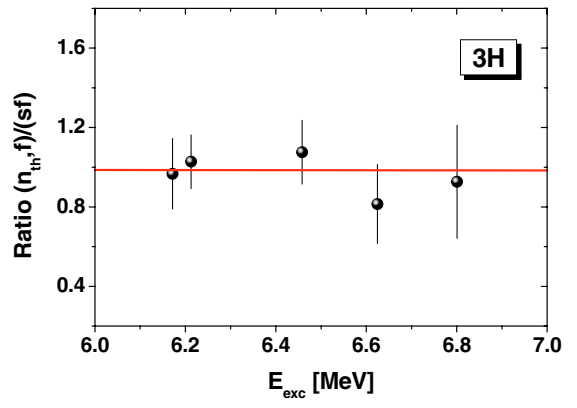

Fig. 5. Ratio of t/B in $(n, f)$ reactions and spontaneous fissions, for a given fissioning nucleus, as a function of excitation energy.

is plotted as a function of the excitation energy. This ratio is compatible with the following law:

$$
(t / B)\left(A_{C N}, E_{\text {exc }}\right) /(t / B)\left(A_{C N}, E_{\text {exc }}=0\right)=1+a_{\text {exc }} E_{\text {exc }}
$$




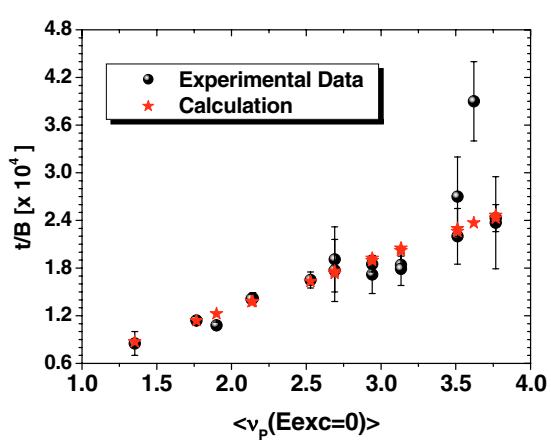

Fig. 6. Comparison between experimental $(t / B)$ data and the $(t / B)$ values calculated from equation (5).

where $(t / B)\left(A_{C N}, E_{\text {exc }}\right)$ is the triton emission probability for a compound nucleus with mass $\mathrm{A}_{C N}$ and having an excitation energy $\mathrm{E}_{\text {exc }}$. The $\mathrm{a}_{\text {exc }}$ parameter is assumed to be independant of the mass of the fissioning nucleus. From the fit performed on the experimental points of figure 5 (straight line), we have found: $\mathrm{a}_{\text {exc }}=-(0.0023 \pm 0.0123) \mathrm{MeV}^{-1}$. In addition, it is generally believed that the energy required to release ternary particles is mainly taken from the available deformation energy of the fissioning nucleus. Since the average prompt neutron multiplicity $\overline{v_{p}}$ is a measure of the deformation energy, a correlation between $\mathrm{t} / \mathrm{B}$ values and $\overline{v_{p}}$ values is expected. In particular, it has been shown already [6] that the spontaneous fission data follow the simple relation:

$$
(t / B)\left(A_{C N}, E_{\text {exc }}=0\right)=a_{\nu} \overline{v_{p}}\left(A_{C N}, E_{\text {exc }}=0\right)
$$

where $\overline{v_{p}}\left(A_{C N}, E_{\text {exc }}=0\right)$ is the average prompt neutron multiplicity for a fissioning nucleus with mass $A_{C N}$ at zero excitation energy. From the fit performed on the spontaneous fission data, we have obtained: $a_{v}=(6.54 \pm 0.22) \times 10^{-5}$. Combining equations (3) and (4), we have:

$$
(t / B)\left(A_{C N}, E_{\text {exc }}\right)=a_{v}\left(1+a_{\text {exc }} E_{\text {exc }}\right) \overline{v_{p}}\left(A_{C N}, E_{\text {exc }}=0\right) .
$$

To evaluate the ternary triton emission probability at a given excitation energy by using equation (5), we need to know the prompt neutron multiplicity $\left(\overline{v_{p}}\left(A_{C N}, E_{e x c}=0\right)\right)$ for spontaneous fission. When no data exist, we have considered the $\overline{v_{p}}\left(A_{C N}, E_{\text {exc }}\right)$ values for the thermal neutron induced fission from which the contribution due to the excitation energy is subtracted:

$$
\overline{v_{p}}\left(A_{C N}, E_{\text {exc }}=0\right)=\overline{v_{p}}\left(A_{C N}, E_{e x c}\right)-\frac{\partial v}{\partial E_{e x c}} E_{e x c} .
$$

Based on a comparison of $\overline{v_{p}}\left(A_{C N}, E_{e x c}=0\right)$ data for spontaneous and thermal neutron induced fission leading to the same compound nucleus, the derivative factor $\partial v / \partial E_{\text {exc }}$ was found to be: $(0.107 \pm 0.005) \mathrm{n} / \mathrm{MeV}$. The $\overline{v_{p}}\left(A_{C N}, E_{\text {exc }}=0\right)$ and $\overline{v_{p}}\left(A_{C N}, E_{e x c}\right)$ data are reported in table 1 . In figure 6 , the comparison between the experimental $(\mathrm{t} / \mathrm{B})$ values and the calculated ones (using eq. (5)) are plotted. An excellent agreement could be reached except for the ${ }^{256} \mathrm{Fm}(\mathrm{sf})$.

\section{Conclusion}

In the present paper, we report new results on the ternary triton characteristics emitted for the ${ }^{249} \mathrm{Cf}\left(\mathrm{n}_{\mathrm{th}}, \mathrm{f}\right)$ and ${ }^{251} \mathrm{Cf}\left(\mathrm{n}_{\mathrm{th}}, \mathrm{f}\right)$ reactions. An updated database, with the energy distribution of the triton particles and their emission probabilities is reported for $(\mathrm{sf})$ decays and $\left(\mathrm{n}_{\mathrm{th}}, \mathrm{f}\right)$ reactions. These characteristics can be very well described from systematics which were revisited. Lastly, a procedure to determine the $(\mathrm{t} / \mathrm{B})$ values as a function of the incident neutron energy is proposed.

Part of this research was performed at the Lawrence Berkeley National Laboratory and the ${ }^{249} \mathrm{Cf}$ isotope was provided by the Office of Sciences, Office of Basic Energy Sciences and the Division of Chemical Sciences, Geosciences, and Biosciences of the US Department of Energy under Contract No. DE-AC02-05CH11231. The authors also acknowledge the assistance of D.K. Shuh of LBNL.

\section{References}

1. O. Serot et al., Proc. Seminar on Fission Pont d'Oye V, Habayla-Neuve, Belgium, 2003, edited by C. Wagemans et al. (World Scientific), p. 151.

2. S. Vermote et al., Proc. 6th Int. Conf. on Dynamical Aspects of Nuclear Fission, Casta-Papiernicka, Slovak Republic, 2006 (World Scientific) (in press).

3. O. Serot, C. Wagemans, Proc. Seminar on Fission Pont d'Oye IV, Habay-la-Neuve, Belgium, 1999, edited by C. Wagemans et al. (World Scientific), p. 45.

4. O. Serot et al., Proc. 5th Int. Conf. on Dynamical Aspects of Nuclear Fission, Casta-Papiernicka, Slovak Republic, 2001 (World Scientific), p. 319.

5. O. Serot, C. Wagemans, Proc. 3rd Int. Conf. on Fission and Properties of Neutron-Rich Nuclei, Sanibel Island, USA, Nov. 2002, edited by J.H. Hamilton et al. (World Scientific), p. 543.

6. C. Wagemans and O. Serot, Proc. 5th Int. Conf. on Dynamical Aspects of Nuclear Fission, Casta-Papiernicka, Slovak Republic, 2001, edited by J. Kliman et al. (World Scientific), p. 301.

7. C. Wagemans, in The Nuclear Fisson Process (CRC Press, Boca Raton, USA, 1991), p. 545.

8. J. Wild et al., Phys. Rev. C 32, 488 (1985).

9. C. Wagemans et al., Phys. Rev. C 33, 943 (1986). 